\title{
General Information Conditioned by a Variable Event
}

\section{Doretta Vivona, Maria Divari}

Department of Basic and Applied Sciences for Engineering, Faculty of Civil and Industrial Engineering, Sapienza

University of Rome, Roma, Italy

Correspondence to: D. Vivona, doretta.vivona@sbai.uniroma1.it, ID: 0000-0002-5267-6497;

M. Divari, maria.divari@alice.it

Keywords: General Information, General Conditional Information, Functional Equations

Received: February 14, $2017 \quad$ Accepted: April 27, $2017 \quad$ Published: April 30, 2017

Copyright () 2017 by authors and Scientific Research Publishing Inc.

This work is licensed under the Creative Commons Attribution International License (CC BY 4.0).

http://creativecommons.org/licenses/by/4.0/

\section{(c) (i) Open Access}

\section{ABSTRACT}

The aim of this paper is to present, by axiomatic way, an idea about the general conditional information of a single, fixed fuzzy set when the conditioning fuzzy event is variable. The properties of this conditional information are translated in a system of functional equations. Some classes of solutions of this functional system have been found.

\section{INTRODUCTION}

In these last years, on crisp setting, measures of information $J$, have been studied by many authors [1] [2-4]. Later particular researches have been done to these information measures defined without probability. The last information measures are called general because they are defined without any probability [5-8]. Analogous studies were presented in [9] on fuzzy setting.

In order to study the integration in information theory without probability, in [10-12] the families $\mathcal{N}, \mathcal{F}, \mathcal{I}_{\infty}, \mathcal{I}_{0}$ have been introduced. The mentioned classes of crisp sets have replaced the families of null sets in the classical theory of integration (i.e. with respect to an additive measure or a probability). A detailed overview can be found in [13].

Later, since 2005, on fuzzy setting, measures of conditional information, when the conditional event is fixed, have been considered and studied (see [14-16]).

Indeed, in this paper, a definition of the general conditional information of a fixed fuzzy set $A$, when the conditioning fuzzy set $H$ is variable will be presented and it will be indicated by $J(A \mid H)$. For this reason, the families mentioned above will be adapted to the fuzzy setting and the corresponding class $\mathcal{H}_{A}$ will be introduced.

Example: let $A$ be the fuzzy set of old men, $H_{1}, H_{2}, H_{3}$ the fuzzy set of those old men, who seem ill, who are ill, who are seriously ill, respectively. These different conditions $H_{1}, H_{2}, H_{3}$ are the conditioning variable events. The conditional information: $J\left(A \mid H_{1}\right), J\left(A \mid H_{2}\right), J\left(A \mid H_{3}\right)$ measure the influence of the grade of the illness in the old men.

The paper is organized in the following way: in Sect. 2 some preliminaires are recalled; in Sect. 3 the 
definition of general information conditioned by a variable event is given. The statement of the problem is presented in Sect. 4 and in Sect. 5 the properties of the form of conditional information are translated in a system of functional equations [17], for which some classes of solutions are shown. Sect. 6 is devoted to the conclusions.

\section{PRELIMINAIRES}

In this paragraph, the definition of general information for fuzzy sets is recalled [9]. The concept of fuzzy set was introduced by Zadeh in [18], for all knowledge see [19, 20].

Let $X$ be an abstract space and $\mathcal{A}$ a-algebra of all fuzzy sets of $X$, such that $(X, \mathcal{A})$ is a measurable space.

Definition 2.1 In the fuzzy setting measure of the general information is a map

$J(\cdot): \mathcal{A} \rightarrow[0,+\infty]$

such that $\forall A_{1}, A_{2} \in \mathcal{A}$ :

(i) $A_{1} \supset A_{2} \Rightarrow J\left(A_{1}\right) \leq J\left(A_{2}\right)$,

(ii) $J(\varnothing)=+\infty, J(X)=0$.

Following the idea presented in $[10,12]$, assigned an information measure $J$, the following families are introduced:

$$
\begin{aligned}
\mathcal{I}_{0} & =\{N \in \mathcal{A} / J(N)=0\} ; \\
\mathcal{I}_{+\infty} & =\{F \in \mathcal{A} / J(F)=+\infty\} .
\end{aligned}
$$

The family (1) is not empty because it contains the whole set $X$ and all supersets $N^{\prime}$ of $N \in \mathcal{I}_{0}$ :

$$
N \in \mathcal{I}_{0}, J(N)=0, \forall N^{\prime} \in \mathcal{A}, N \subset N^{\prime}, J\left(N^{\prime}\right) \leq J(N)=0 \Rightarrow N^{\prime} \in \mathcal{I}_{0} .
$$

$\mathcal{I}_{0}$ is not an ideal $[21,22]$ because it is not stable with respect to the union between fuzzy sets.

The family (2) is not empty because it contains the empty set $\varnothing$ and all subsets $F^{\prime}$ of $F \in \mathcal{I}_{+\infty}$ :

$$
F \in \mathcal{I}_{+\infty}, J(F)=+\infty, \forall F^{\prime} \in \mathcal{A}, F^{\prime} \subset F, J(F) \geq J\left(F^{\prime}\right)=+\infty \Rightarrow F^{\prime} \in \mathcal{I}_{+\infty} .
$$

$\mathcal{I}_{+\infty}$ is not an filter $[21,22]$ because it is not stable with respect to the intersection between fuzzy sets.

\section{MEASURE OF GENERAL CONDITIONAL INFORMATION BY A VARIABLE EVENT}

From now on, the family $\mathcal{H}=\mathcal{A}-\mathcal{I}_{+\infty}$ shall be considered and measure of general conditional information of a fixed fuzzy set $A \in \mathcal{A}, J(A \mid \cdot)$ defined on the family $\mathcal{H}$ will be introduced.

Definition 3.1 Measure of general information of a fixed $A \in \mathcal{A}$, conditioned by a variable event $H \in \mathcal{H}$ is a map

such that

$$
J(A \mid \cdot): H \rightarrow[0,+\infty]
$$

(j) $H \supset H^{\prime} \Rightarrow J(A \mid H) \leq J\left(A \mid H^{\prime}\right), \forall H, H^{\prime} \in \mathcal{H}$,

(jj) $J(A \mid N)=J(A)$ if $N \in \mathcal{I}_{0}$.

From the previous axioms, it follows that $J(A \mid X)=J(A)$.

The condition (j) is the monotonicity, the (jj) means that all null sets $N \in \mathcal{N}_{0}$ don't condition any fuzzy set $A \in \mathcal{A}$.

\section{STATEMENT OF THE PROBLEM}

Taking into account the previous axiomatic statement, fixed an information measure $J$ on $\mathcal{A}$ and any fuzzy set $A \in \mathcal{A}$, some classes of measures $J(A \mid H)$, will be sought by supposing that $J(A \mid H)$ de- 
pends only on $J(A), J(H)$ and $J(A \cap H)$. Now it is necessary to specify the family where $H$ belongs.

Fixed $A$, our definition is restricted to the following family

$$
\mathcal{H}_{A}=\{H \in \mathcal{H} / J(A \cap H) \neq+\infty\}
$$

at least $\mathcal{H}_{A}$ contains the whole space $X$, so this family is not empty. The condition $J(A \cap H) \neq+\infty$ ensures that $J(H) \neq+\infty$ : in fact if $J(H)=+\infty$ from the monotonicity of $J$, it is $J(A \cap H)=+\infty$.

So, the information $J(A \mid \cdot)$ is the function

expressed by a function $\Phi: V \rightarrow(0,+\infty)$, such that

$$
J(A \mid \cdot): \mathcal{H}_{A} \rightarrow[0,+\infty]
$$

$$
J(A \mid H)=\Phi(J(A), J(H), J(A \cap H))
$$

with

$$
V=\{(x, y, z) / x=J(A), y=J(H), z=J(A \cap H), x, y, z \in[0,+\infty], x \leq z, y \leq z\} .
$$

This justifies the domain of the function $\Phi$.

From (3), (j), (jj), $\forall H_{1}, H_{2} \in \mathcal{H}$, the function $\Phi$ shall satisfy the following properties:

(I) $H_{1} \supset H_{2} \Rightarrow \Phi\left(J(A), J\left(H_{1}\right), J\left(A \cap H_{1}\right)\right) \leq \Phi\left(J(A), J\left(H_{2}\right), J\left(A \cap H_{2}\right)\right)$,

(II) $\Phi(J(A), J(N), J(A \cap N))=J(A), \forall N \in \mathcal{I}_{0}$.

Setting $J(A)=x, J\left(H_{1}\right)=y_{1}, J\left(H_{2}\right)=y_{2}, J\left(A \cap H_{1}\right)=z_{1}$,

$J\left(A \cap H_{2}\right)=z_{2}, J(A \cap N)=t$ with $x, y_{1}, y_{2}, z_{1}, z_{2} \in[0,+\infty)$ and $x \leq z_{1}$,

$y_{1} \leq z_{1}, x \leq z_{2}, y_{1} \leq z_{2}, x \leq t$, the following system of functional equations is obtained:

$$
\left\{\begin{array}{l}
(1) \Phi\left(x, y_{1}, z_{1}\right) \leq \Phi\left(x, y_{2}, z_{2}\right), \quad x \leq z_{1}, y_{1} \leq z_{1}, x \leq z_{2}, y_{2} \leq z_{2} \\
(2) \Phi(x, 0, t)=x, \quad x \leq t .
\end{array}\right.
$$

\section{SOLUTION OF THE PROBLEM}

A function $\Phi$ continuous defined in the following set:

$$
D=\{(x, y, z): x, y, z \in[0,+\infty), x \leq z, y \leq z\}
$$

will be sought as an universal law in the sense that the equation and the inequality about the function of the system must be satisfied for all values and variables in their proper space, which satisfies the system [(1)-(2)].

Now, the following results are proved:

Proposition 4.1 A class of solutions of the system [(1)-(2)] is:

$$
\Phi_{h}(x, y, z)=h^{-1}([h(x)+h(y)] \wedge h(z)),
$$

where $h:[0,+\infty) \rightarrow[0,+\infty)$ is any continuous function, strictly increasing with $h(0)=0$.

Proof. The condition (1) follows by the monotonicity of the function $h$. The second one (2) results from the value $h(0)=0$. and the property of the function $h$.

Proposition 4.2 A class of solutions of the system [(1)-(2)] is:

$$
\Phi_{h}(x, y, z)=h^{-1}([h(x) \vee h(y)] \wedge h(z))=h^{-1}(h(x) \vee h(y)),
$$

where $h:[0,+\infty) \rightarrow[0,+\infty)$ is any continuous function, strictly increasing with $h(0)=0$.

Proof. The proof is immediate.

Proposition 4.3 A class of solutions of the system [(1)-(2)] is:

$$
\Phi_{h}(x, y, z)=h^{-1}([h(x) \wedge h(y)] \vee h(z))=z,
$$


where $h:[0,+\infty) \rightarrow[0,+\infty)$ is any continuous function, strictly increasing with $h(0)=0$.

Proof. The proof is immediate.

From (4) (5) and (6), the following expressions of conditional information have been obtained, respectively:

$$
\begin{gathered}
J_{h}(A \mid H)=h^{-1}([h(J(A))+h(J(H))] \wedge h(J(A \cap H))) ; \\
J_{h}(A \mid H)=h^{-1}(h(J(A))) \vee h(J(H)),
\end{gathered}
$$

where $h:[0,+\infty) \rightarrow[0,+\infty)$ is any continuous function, strictly increasing with $h(0)=0$, and

$$
J(A \mid H)=J(A \cap H) .
$$

\section{CONCLUSIONS}

In this paper, for the first time, we present an axiomatic definition of the information $J(A \mid H)$, when the conditioning event is variable.

We think that this axiomatic approach could be useful for future applications.

\section{ACKNOWLEDGMENTS}

For the first author, this research is in the framework of GNFM of MIUR (Italy) and University "Sapienza" Roma.

The authors declare that they have no conflict of interest.

\section{REFERENCES}

1. Shannon, C. and Weaver, W. (1949) The Mathematical Theory of Communication. University of Illinois Press, Urbana.

2. Khincin, A.Y. (1957) Mathematical Foundation of Information Theory. Dover Publication, New York.

3. Rényi, A. (1961) On Measures of Entropy and Information. Proceedings IV Berkeley Symposium on Mathematical Statistics and Probability, Berkeley, 20-30 June 1961, Vol. 1, 547-561.

4. Aczél, J. (1969) Probability and Information Theory. Lectures Notes in Mathematics, Vol. 89, Springer-Verlag, Berlin, 1-11.

5. Kampé de Fériet, J. and Forte, B. (1967) Information et Probabilité. Comptes Rendus de P Académie des Sciences Paris, 265, 110-114, 142-146, 350-353.

6. Forte, B. (1969) Measure of Information. The General Axiomatic Theory. R.I.R.O., Informatique Théorique et Applications, R3, 63-90.

7. Kampé de Fériet, J. and Benvenuti, P. (1969) Sur une classe d'informations. Comptes Rendus de P Académie des Sciences Paris, 269, 97-101.

8. Kampé de Fériet, J. (1970) Mesures de l'information fornie par un evénement. Colloque International Du Centre National de la Recherche Scientifique, 186, 191-221.

9. Benvenuti, P., Vivona, D. and Divari, M. (1990) A General Information for Fuzzy Sets. In: Bouchon-Meunier, B., Yager, R.R. and Zadeh, L.A., Eds., Uncertainty in Knowledge Bases, IPMU 1990, Lecture Notes in Computer Science, Vol. 521, Springer, Berlin, Heidelberg, 307-316. https://doi.org/10.1007/BFb0028117

10. Vivona, D. (1982) L'informazione integrale. Quaderno. 19 Istituto di Matematica Applicata delle Facoltá di Ingeneria, Universitá di Roma, 245, 3-15.

11. Benvenuti. P., Vivona, D. and Divari, M. (1988) Sull'Integrale nella Teoria dell'Informazione. Rendiconti di 
Matematica di Roma, 8, 31-43.

12. Vivona, D. and Divari, M. (1989) Sull'entropia integrale nella teoria dell'informazione. Rendiconti di Matematica di Roma, 9, 145-159.

13. Vivona, D. (2004) Information Theory and Fuzzy Integrals: Along the Way Paved by Pietro Benvenuti. Proceedings of IPMU04, Perugia, 4-9 July 2004, 1957-1963.

14. Vivona, D. and Divari, M. (2005) On a Conditional Information for Fuzzy Sets. Proceedings of AGOP05, Lugano, 10-15 July 2005, 147-149.

15. Bouchon, B., Coletti, G. and Masala, C. (2006) A General Theory of Conditional Decomposable Information Measures. Proceedings IPMU06, Paris, 2-7 July 2006, Vol. 1, 97-104.

16. Vivona, D. and Divari, M. (2008) Aggregation Operators for Conditional Information without Probability. Proceedings IPMU08, Malaga, 22-27 June 2008, 258-260.

17. Aczél, J. (1966) Lectures on Functional Equations and Their Applications. Academic Press, New York.

18. Zadeh, L.A. (1965) Fuzzy Sets. Information and Control, 8, 338-353.

19. Klir, G.J. and Folger, T.A. (1988) Fuzzy Sets, Uncertainty, and Information. Prentice Hall, Upper Saddle River.

20. Boskov, B. (1993) Fuzzy Thinking: The New Science of Fuzzy Logic.

21. Halmos, P.R. (1969) Measure Theory. Van Nostrand Reinhold Company, New York.

22. Yosida, K. (1995) Functional Analysis. Springer, Berlin. https://doi.org/10.1007/978-3-642-61859-8

\section{Scientific Research Publishing}

\section{Submit or recommend next manuscript to SCIRP and we will provide best service for you:}

Accepting pre-submission inquiries through Email, Facebook, LinkedIn, Twitter, etc.

A wide selection of journals (inclusive of 9 subjects, more than 200 journals)

Providing 24-hour high-quality service

User-friendly online submission system

Fair and swift peer-review system

Efficient typesetting and proofreading procedure

Display of the result of downloads and visits, as well as the number of cited articles

Maximum dissemination of your research work

Submit your manuscript at: http://papersubmission.scirp.org/

Or contact ns@scirp.org 